\title{
A Vaccine for Syphilis
}

\author{
Stewart Sell MD* \\ Department of Health, Wadsworth Laboratory, USA
}

Received: 㭗: July 23, 2018; Published: 狦 July 27, 2018

*Corresponding author: Stewart Sell, Department of Health, Wadsworth Laboratory, USA, Empire State Plaza Albany.

\begin{abstract}
Design and application of a vaccine for syphilis is proposed. Syphilis, once a widespread and crippling disease, has decreased in prevalence and significance due to public health education and the use of penicillin, which is extremely effective in curing the disease. This decline has reduced the urgency for a vaccine for syphilis. However, in recent years the incidence of syphilis has been increasing. A persistent reservoir remains in highly impacted groups such as sex workers and men who have sex with men. It is proposed that the critical application of a vaccine for syphilis to these groups could eventually eliminate this disease. Evidence is presented that the most effective immune response to syphilis infection is delayed type hypersensitivity (DTH). In this review a design for a vaccine for syphilis directed to the induction of DTH is described.
\end{abstract}

Abbreviations: DTH: Delayed Type Hypersensitivity; TPRK: Treponema Pallidum Repeat Protein K; BCG: Bacille-Calmette-Guerin; APH: Aminoglycoside Phosphotransferase; ORIE: Origin of Replication; AATP: Attachment Site and the Gene Encoding the Integrase Protein

\section{Introduction}

Syphilis has a long and devastating history. It first appeared in Europe shortly after the return of Columbus' ships from the new world. Treatises by Francisco Lopez de Villalobos in 1498 [1] and Ray Diaz de Isla in 1539 [2] accurately describe the clinical symptoms and epidemiology as syphilis jumped form the new world to the old world. The disease spread rapidly throughout Europe because of the dispersal of infected soldiers after the conquest of Naples by Charles VIII in 1494. Although some questions have been raised that there might have been a form of syphilis in the old world before this, this idea has largely been discredited [3]. In addition, at that time syphilis was spreading through Europe, sexual behavior was not yet restricted by religious beliefs. In fact, D. H. Lawrence attributed the rise of puritanism and its sexual restrictions in Europe to the arrival of syphilis [4]. In the early 20th century Syphilis was considered one of the three great plagues, along with tuberculosis and cancer [5]. An extensive investigation by The Royal Commission on Venereal Diseases in England and Wales in 1916 concluded that the number of persons with syphilis "cannot fall below 10 per cent of the whole population...", [6] and more than $20 \%$ of patients in U.S. mental hospital in the 1920s had tertiary syphilis [7].

Syphilis occupied a prominent position in the minds of clinicians of the time not only because of the prevalence but also because of the wide range of presentation of signs and symptoms. Sir William Osler, recognized as the preeminent physician of his time, noted that syphilis was the great imitator and that "he who knows syphilis knows medicine" [8]. He noted that syphilis struck terror into the hearts by the rapidity of its spread, the ravages it made, and the apparent helplessness of the physicians to cure it.
Although vaccines had been effectively applied to other infectious diseases, no one appeared to have an idea how to make a vaccine for syphilis. Then Interest I should be lc in a vaccine for syphilis declined as the prevalence of the disease decreased. The incidence of syphilis first declined because of extensive public education efforts in the mid- $20^{\text {th }}$ century, but was even more affected by the introduction of penicillin. Penicillin is an extremely effective treatment for syphilis and the use of penicillin to treat syphilis resulted in a marked decline in the prevalence of the disease. Between 1940 and 1951 the incidence of syphilis dropped from close to 400 cases per 100,000 to less than 100 per 100,000 in 1950 . In 2000 the national number of reported primary and secondary syphilis was 2.1 cases per 100,000 population. However, the rate has increased almost every year since then. In 2016 the rate increased to 8.7, the highest seen since 1993 [9].

Recently an argument for the need of a vaccine for syphilis has been made [10]. It is unlikely that syphilis vaccination would be applied to the general population. However, it could be very applicable to selected populations, for example: sex workers and men who have sex with men. Up to $5 \%$ of sex workers in 20 of 45 reporting countries are infected with syphilis [11]. Thus, prevention of syphilis in sex workers by vaccination could by itself greatly reduce spread of infection, particularly in vulnerable populations. In the United States over $50 \%$ of diagnosed cases of primary or secondary syphilis are in men who have sex only with men [9]. Thus, this population would also be a prime target for vaccination. In addition, 1 million pregnant women world-wide have active syphilis infection and are at risk for adverse pregnancy 
outcomes such as stillbirth and neonatal death due to congenital syphilis. Congenital syphilis has increased by $27.6 \%$ between 2015 and 2016 [9]. Selective immunization of women at high risk for syphilis and pregnancy should also be considered.

\section{Immunodefense Against Syphilis}

To construct a useful vaccine for an infectious disease one must know the type of immune response that is effective in combatting the disease [12]. Studies in experimental infection of rabbits with syphilis have resulted in better understanding of the protective immune response to syphilis infection. In fact, the primary chancre of syphilis in humans grossly and microscopically is very similar to that of experimental infection of the skin of rabbits [13] (Figure 1). In experimental infection of the testes of rabbits with the causative agent of syphilis, Treponema pallidum, organisms can be identified by fluorescence microscopy in the connective tissue within 3 days of inoculation. The number of organisms increases exponentially in the interstitial connective tissue until about 11-12 days after infection; then within a few days the organisms disappear [14,15]. This sequence is accompanied by massive swelling of the testes to about twice normal size, infiltration of the infected tissue with T-cells and then macrophages which rapidly phagocytose and digest large number of the organisms (Figure 2).

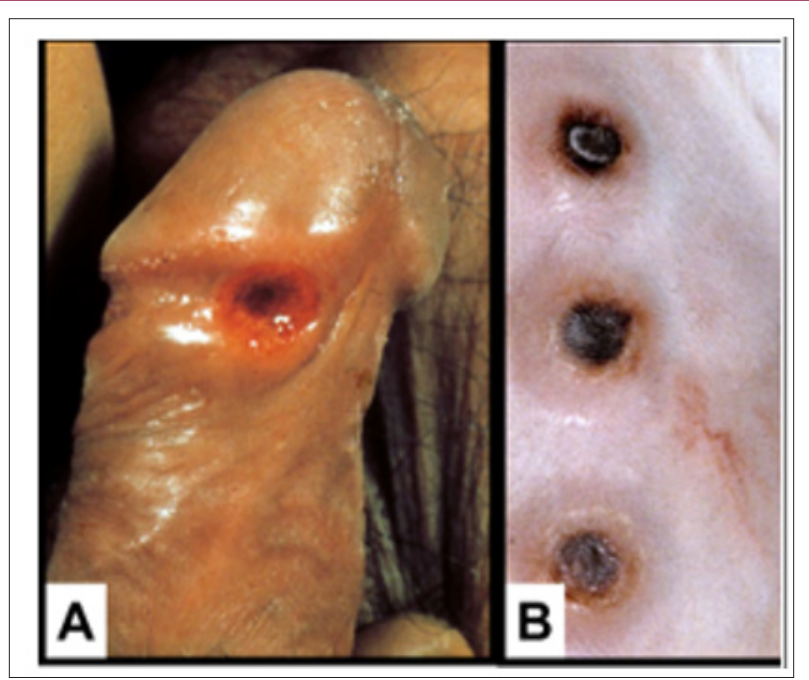

Figure 1: A. Human syphilitic chancre and Syphilitic skin lesions in infected rabbits. Both are DTH reactions.

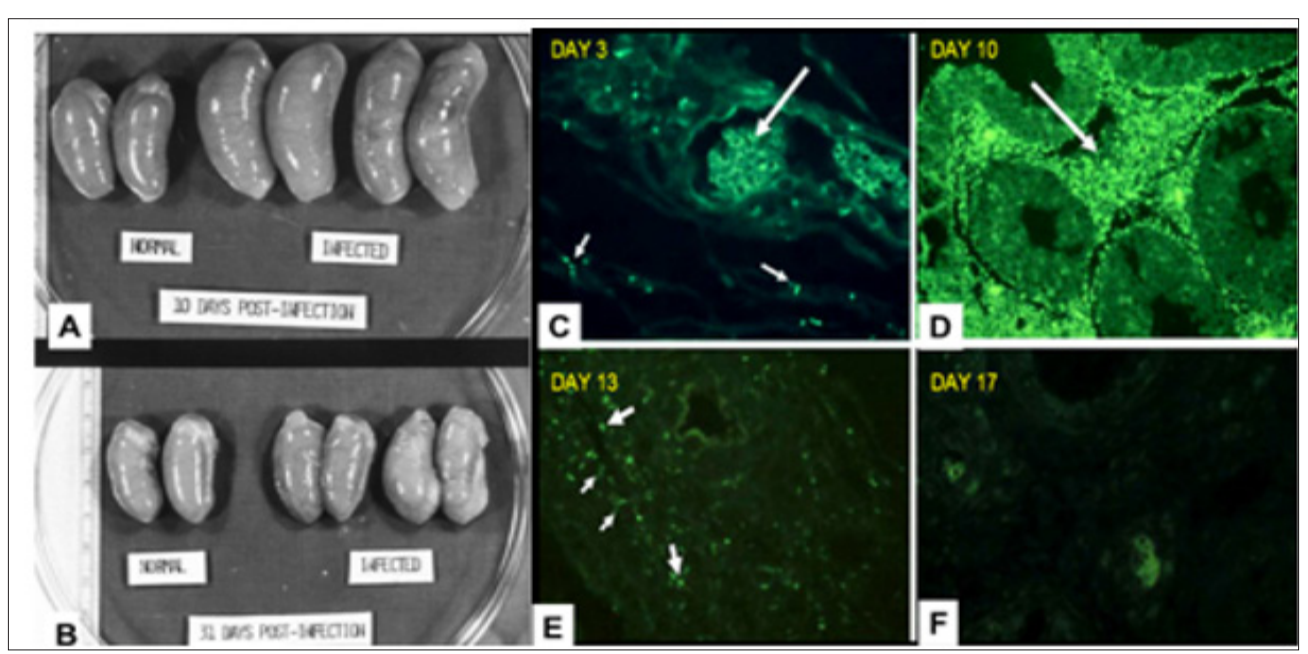

Figure 2: A,B Gross photos of testes; C-F Immunofluorescence of T. pallidum in testes during infection. A. On day 10 after infection testes are essentially twice normal size. B. On day 30 after infection testes have returned to only slightly larger than normal. C. 3 days after infection a few organisms (small arrows) are present in interstitial tissue. Large arrow point to autofluorescent red blood cells. D. 10 days after infection the interstitial tissue is filled with T. pallidum. E. 13 days after infection only a few organisms (small arrows) can be identified and there is fluorescence of fragments, presumably in macrophages.. F 17 days after infection no organisms can be see and only fluorescent smudges remain.

Electron microscopy of the active infective stage shows the serpentine-like T. pallidum "swimming" in interstitial connective tissue. During the active clearing of the infection partially disrupted organisms can be seen within macrophages [16] (Figure 3). A similar reaction occurs after infection in the skin. This reaction correlates with hyperplasia of the diffuse cortex (T-cell zone) of the draining lymph nodes [17], histologic evidence of a T-cell response. Thus, it is concluded that the primary chancre of syphilis is, in fact, a 


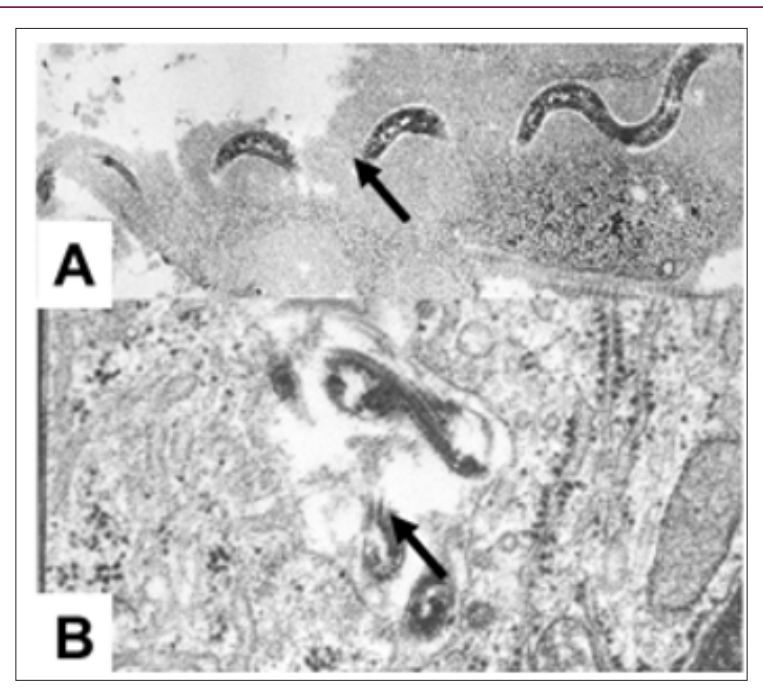

Figure 3: Electron microscopic photos of T. pallidum. A. In interstitial connective tissue during active infection. B. In phagosomes of a macrophage during clearing phase. Arrows point to lateral filaments of T. pallidum.

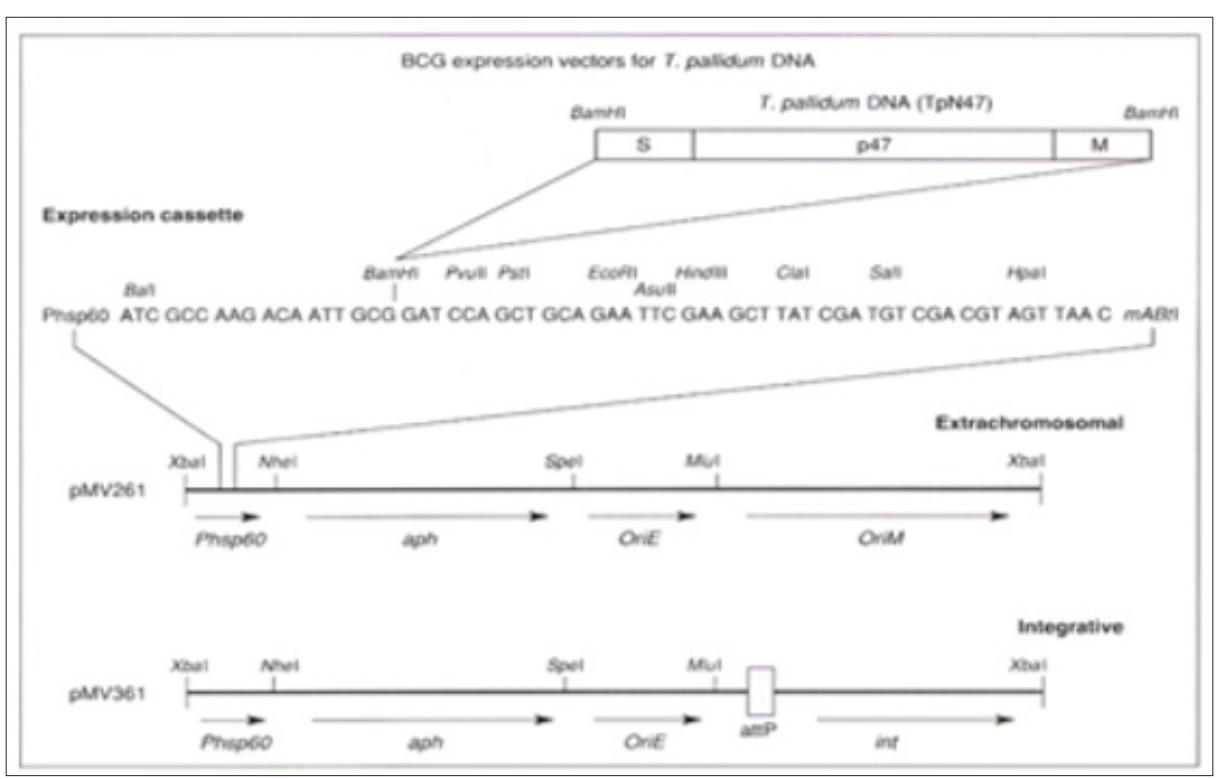

Figure 4: Proposed insertion of DNA sequence for T. pallidum antigen TpN47 into BCG. From ref. 12. See text.

delayed type hypersensitivity (DTH) reaction, that is exquisitely effective in curing the infection [13]. Secondary and tertiary infection may occur when organisms spread to "protected sites" or when the DTH response is inadequate and antibody production predominates [12]. In fact, the role of antibody and DTH in syphilis may be compared to that in leprosy [12]. In leprosy a strong DTH response (Tuberculoid Leprosy) is associated with arrested disease, whereas a strong antibody response is associated with prolonged and progressive infection (Lepromatous Leprosy) [18]. Similarly, in syphilis a strong DTH response correlates with limitation to a primary lesion (chancre) whereas a weak DTH response is associated with secondary and tertiary disease [13]. Thus, we concluded that a vaccine for syphilis should be designed to induce DTH. An effective vaccine for inducing DTH to T. pallidum should selectively deliver the immunogen to interdigitating reticulum cells for presentation in association with MHC class II antigens to paracortical CD4+ TDTH precursor cells [12].

\section{T. Pallidum Immunization}

A time-honored clinical observation is that patients that have recovered from a primary syphilis infection demonstrate what is called "chancre immunity [19]. Previously infected rabbits with healed chancres also demonstrate chancre immunity [20], i.e., rabbits which develop and heal a primary lesion (chancre) are now resistant to a second infection. In a related observation, if patients with syphilis are successfully treated with penicillin before a chancre has healed they will not be resistant and may be re-infected, so-called "ping-pong" syphilis [21]. If infection is eliminated before full DTH is established, the individual has not developed protective immunity. Thus, in both humans and rabbits, successful induction of delayed hypersensitivity to syphilis results in long-lasting protection against re-infection. Attempts to induce protective immunity using avirulent treponemes, attenuated or heat-inactivated organisms, or extracts of pathogenic organisms 
have been only partially successful and in only a few instances 10). Miller obtained complete long-term protection following a 37 week long, 60 intravenous immunization, schedule, using large doses of gamma-irradiated organisms [22]. The requirement to grow large numbers of organisms in rabbit testicles to obtain immunizing antigens renders such protocol impractical for other than experimental use. Further attempts at immunization have been attempted using selected antigen of T. pallidum (Tp).

\section{T Pallidum Antigens}

Over 40 T. Pallidum antigen are available commercially. Including $\mathrm{T}$. pallidum TpN47, $\mathrm{T}$. pallidum major membrane immunogens Tp15, Tp17 and Tp0453. Although antibodies to the variable regions of Treponema pallidum repeat protein $\mathrm{K}$ (TPRK) correlate with protection against infection [23], in general immunizations that induce antibody and not DTH are ineffective. Tomson et al immunized rabbits with Tp0155, Tp0326, Tp0326, and Tp0956 and although high antibody titers were obtained, the immunized rabbits did not demonstrate protection against infection [24]. This confirms many studies that show that although immunoglobulin antibodies may enhance macrophage killing of T. pallidum (opsonization) [25], humoral antibodies are relatively ineffective in preventing syphilis infection [26]. Thus, we proposed using modified BCG to induce DTH to T. pallidum as a potential effective vaccine [12].

\section{BCG Vector for Syphilis Vaccine}

According to the hypothesis presented above, selection of immunogen presentation for syphilis vaccination should be directed to induction of DTH. There are four proposed antigen processing pathways:

a) Exogenous presentation via follicular dendritic cells to Th2 helper cells for IgE/IgA production to helminths.

b) Exogenous presentation via follicular dendritic cells to Th1 helper cells for IgM/IgG antibody to bacteria.

c) Presentation by interdigitating reticulum cells to Th1 helper cells to induce DTH to mycobacteria.

d) Endogenous antigen processing by any nucleated cells to generate T-cytotoxic cells to viruses [12].

Many antigens expressed by vaccinia virus vectors have generated strong cell-mediated immune responses [27], but this approach whereby antigens are presented by infected epithelial cells is effective for inducing a T-cytotoxic response and not necessarily DTH [12]. To induce DTH we propose using BCG (Bacille-CalmetteGuerin) vectors [28]. Thus, rather than presenting antigens by the endogenous pathway, interdigitating reticulum cells in the cortex of lymph nodes would be presenting antigen To Th1 helper cells by the exogenous pathway.

In experimental animals, Freund's adjuvant is effective in inducing DTH, but this is too toxic for human use. BCG is a live attenuated bovine tubercle bacillus approved for selective human use for vaccination against TB. It is effective in inducing specific TDTH cells which in the presence of antigen activate macrophages. Although now more limited in use, BCG is approved for human use and has been applied extensively in the past with a low incidence of serious complications. It is inexpensive to produce and can produce long term immunity. Since the Tp antigens listed above are recombinant proteins, the encoding DNA sequences are readily available for insertion into the proposed BCG vector. BCG vectors for producing such vaccines have been previously designed and constructed [29-31]. The procedure for the process using TpN47 is shown in Figure 4, reproduced from reference [12]. The extrachromosomal (PMV262) and integrative (PMV361) BCG expression vectors of BCG are available for cloning T. pallidum DNA.

Both plasmids contain the mycobacterium heat shock protein promoter (Phsp60), multiple sites for inserting DNA, a transcriptional terminator (mAbt1), the transposon Tn903-derived aminoglycoside phosphotransferase (APH) gene which confers kanamycin-resistance as a selection process, and an origin of replication (ORIE) for propagation in E. Coli. pMV261 also contains mycobacterial origin of replication (ORIM) and thus can propagated extra-chromosomally in BCG. PMV361 contains phage attachment site (AATP) and the gene encoding the integrase protein (int). The PMV 361 plasmids replicated in BCG by integration into the BCG genome through site-specific recombination and replication as part of the BCG chromosome [31]. This proposal was originally made in 1993 [12], and although there is a well worked out experimental model to test a vaccine for syphilis, funding has not been forthcoming to implement it [10].

\section{References}

1. Lopez de Villalobos R (1890) On the plague of the cursed glands. 1498, French Translation: B. Masson, Ed Paris.

2. Diaz de Isla R (1539) The serpentine disease. Con priuioegio imperial y del rey de Portugal.

3. Firth J Syphilis (2012) Its early history and treatment until penicillin and the debate on its origins. J Military and Veterans' Health 20: 49-58.

4. Andreski S (1982) The Syphilitic Shock: A new explanation of the "Great Witch Craze" of the $16^{\text {th }}$ and $17^{\text {th }}$ centuries in the light of medicine and psychiatry. Encounter 55: 76-81.

5. Stokes JH (1920) The third great plague. A discussion of syphilis for everyday people, WB. Saunders Co. Philadelphia and London, UK.

6. Szreter S (2014) The prevalence of syphilis in England and Wales on the eve of the great war: revisiting the estimates of the Royal Commission on "Venereal Diseases, 1913-1916. Social History of Med. 27: 508-529.

7. Brant AM (1987) No magic bullet: A social history of Venereal disease in the United States Since 1980. Oxford U Press, New York, USA.

8. Osler W McCrea T (1921) The principles and practice of medicine: Designed for the use of practioners and students of medicine. D Appleton and Co. New York and London, USA.

9. (2016) Center for Disease Control and Prevention. Sexually Transmitted Diseases Syphilis.

10. Cameron CE, Lukehart SA (2014) Current status of syphilic vaccine development: Need, challenges, prospects. Vaccine 32: 1602-1609.

11. (2016) World Health Organization, Global Health Observatory.

12. Sell S, Hsu PL (1993) Delayed hypersensitivity, immune deviation, antigen processing and T-helper cell subset selection in syphilis pathogenesis and vaccine design. Immunol. Today, 14: 576-582.

13. Carlson JA, Dabri G, Cribier B, Sell S (2011) The immunopathobiology of syphilis. The manifestations and course of syphilis are determined by the level of delayed hypersensitivity. Am J Dermatopathol 33: 433-460. 
14. Lukehart SA, BakerZander SA, Lloyd RM, Sell S (1979) Characterization of lymphocyte responsiveness in early experimental syphilis II. Nature of cellular infiltration and Treponema pallidum distribution in testicular lesions. J Immunol 14: 461467.

15. BakerZander S, Sell S (1980) A histopathologic study of the course of syphilis in the experimentally infected rabbit: Demonstration of long lasting cellular immunity. Am J Pathol 101: 387414.

16. Sell S, BakerZander S, Powell HC (1982) Experimental syphilitic orchitis in rabbits. Ultrastructural appearance of T. pallidum during phagocytosis and dissolution by macrophages in vivo. Lab Invest 46: 355364.

17. Sell S, BakerZander S, Lloyd RM (1980) T-cell hyperplasia of lymphoid tissues in rabbits infected with T. pallidum: a preliminary report showing no evidence for immune suppression. Sexually Transmitted Diseases 7: 7480 .

18. Turk JL (1967) Delayed Hypersensitivity. New York Wiley, USA.

19. Neisser A (1964) Beitrange zur Pathologie und Therapie der syphilis, Springer, Berlin.

20. Cannefax GR (1964) Immunity in syphilis. Brit J vener Dis 41: 260-274.

21. Schoch AG, Alexander LJ (1949) Reinfection and relapse after treatment of early syphilis with penicillin: analysis of 137 cases of infections failure in a totoal series of 1,105 cases. Arch Derm Shphilil 60: 690-700.

22. Miller JN (1973) Immunity in experimental syphilis. VI. Successful vaccination of rabbits with Treponema pallidum Nichols strain, attenuated by x-irradiation. J Immunol 110: 1206-1215.

\section{ISSN: 2574-1241}

DOI: $10.26717 / B J S T R .2018 .07 .001486$

Stewart Sell. Biomed J Sci \& Tech Res

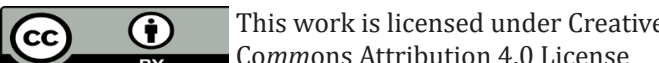

Submission Link: https://biomedres.us/submit-manuscript.php
23. Morgan CA, Lukehart SA, Van Voorhis WC (2003) Protection against syphilis correlates with specificity of antibodies to the variable regions of Treponema pallidum repeat protein K. Infect Immun. 71: 5605-5612.

24. Tomson FL, Conley PG, Norgard MV, Hagman KE (2007) Assessment of cell-surface exposure and vaccinogenic potentials of Treponema pallidum candidate outer membrane proteins. Microbes Infect. 9: 12671275.

25. Baker-Zander SA, Lukehart SA (1992) Macrophage-mediated killing of opsonized Treponema pallidum. J Infect Dis 165: 69-74.

26. Bishop NH, Miller JN (1976) Humoral immunity in experimental syphilis I. The demonstration of resistance conferred by passive immunization. J Immunol 117: 191-196.

27. Mackett M, Smith G (1986) Vaccinia virus expression vectors. J Gen Virol 10: 2067-2082

28. Kubanov A, Runia A, Deryabin D (2017) Novel Treponema pallidum recombinant antigens for syphilis diagnostics: Current status and future prospects. Biomed Res Int PMID 28523273.

29. Bloom BR (1989) New approaches to vaccine development. Rev Infect 11(2): 460-510

30. Snapper SB, Lugosi J, Jekkel A, Melton RE, Kieser T, et al. (1988) Lysogeny and transformation in mycobacteria: stable expression of foreign genes. Proc Natl Acad Sci USQA 85: 6987-6991.

31. Stover CK, de la Cruze VF, Fuerst TR, Burlein JE, Benson LA, et al. (1991) New use of BCG for recombinant vaccines. Nature 351: 456-460.

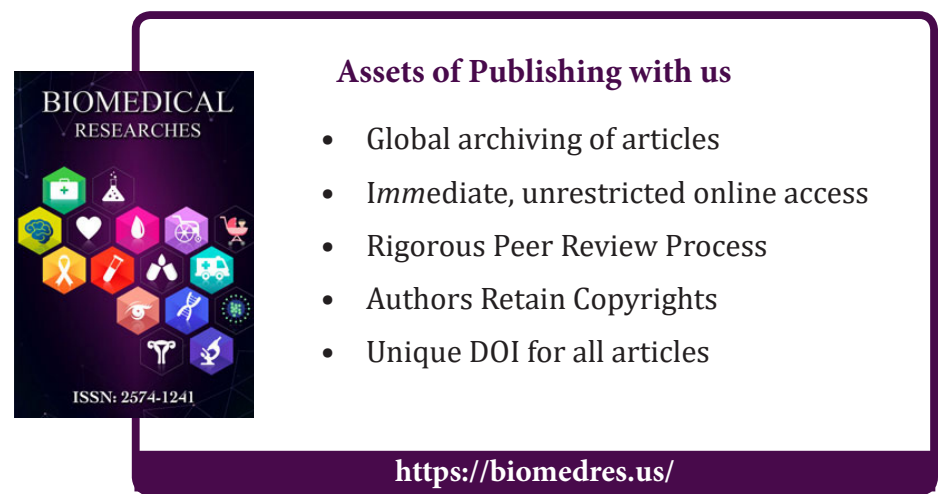

